\title{
Controle de sistemas não-linares utilizando a técnica de identificação de controladores
}

\author{
Alexandre Molter, $\quad$ Fabrício Bandeira Cabral, \\ Departamento de Matemática e Estatística, Universidade Federal de Pelotas, \\ Campus Universitário, 354, 96010-900, Pelotas, RS. \\ E-mail: alexandre.molter@ufpel.edu.br, fabriciocabral2000@yahoo.com.
}

\begin{abstract}
Resumo: Neste artigo aplicamos a técnica de identificação de controladores ao controle de um sistema não-linear sem o conhecimento dos valores dos parâmetros e das funções de controle. Propomos uma classe de controladores proporcionais como candidatos a controlar o sistema de modo que este siga trajetórias desejadas. Simulações e resultados são apresentados. O artigo também tem caráter didático ao mostrar de forma simples como aplicar a técnica de identificação de controladores.
\end{abstract}

Palavras-chave: sistemas não-lineares, controle, identificação de controladores.

\section{Introdução}

O controle de sistemas não-lineares, mediante diferentes técnicas e metodologias, continua sendo de grande interesse de pesquisadores em diversas áreas do conhecimento. Diversas teorias de controle estão baseadas em controladores PIDs. Além disso, os controladores são, na maioria das vezes, ajustados por métodos empíricos e operadores experientes. Em paralelo a esta situação temos desenvolvimentos teóricos complexos e com muitas hipóteses restritivas sobre os sistemas a serem controlados.

A busca de métodos de controle que usem informações minimais sobre os sistemas a serem controlados deu origem aos métodos de identificação de contoladores e de controle não-falsificado $[4,5]$. Note-se que estes métodos fazem parte de um questionamento mais amplo da formulação do problema de controle [6]. A vantagem destes métodos em relação a outros é que eles não necessitam, para sua aplicação, conhecimentos prévios do estado, propriedades físicas dos modelos ou funções de controle.

O objetivo neste trabalho é utilizar a técnica de identificação de controladores para identificar funções de controle e controlar a trajetória de um sistema dinâmico não-linear bem conhecido, a saber, o sistema de Rössler [3]. Para controlar este sistema já foram aplicadas diversas metodologias, dentre as quais, as utilizadas em [1] e [2]. Em geral, o que se percebe nestas aplicações é que os controladores utilizados são de controle proporcional. Então, a idéia é propor funções de controle que sejam simples, como o as do controle proporcional. Se identifica, dentre uma família de candidatos a controladores, aqueles que apresentam melhor desempenho. Além disto o fator de proporcionalidade é modificado dentro de certo período de tempo previamente estipulado. Esta modificação tem o intuito de melhorar o desempenho do controlador.

Para verificar a eficiência da metodologia proposta serão apresentadas simulações do sistema de Rössler, onde se busca levar as trajetórias temporais do sistema para trajetórias desejadas.

\section{A técnica de Identificação de Controladores}

A técnica de identificação de controladores está descrita de forma generalizada em [4]. Nesta técnica não são utilizados modelos para o sistema a ser controlado, a não ser o próprio modelo 
fornecido pelos dados experimentais. No caso em estudo, necessita-se apenas de funções de referência $r$ para obter as funções de controle $u$ (neste estudo $u$ são leis de controle proporcional) e os dados adivindos do sistema dinâmico $y$. Propõe-se uma família de controladores candidatos e avalia-se o seu desempenho. Utilizando-se o conceito de referência fictícia podemos avaliar o desempenho de controladores que não estão atuando sobre a planta no momento.

Ilustraremos esta técnica para o caso de uma família de controladores proporcionais. A lei de controle será dada por

$$
u=K(r-y),
$$

donde

$$
u / K=(r-y)
$$

e, consequentemente a referência fictícia será dada por

$$
r_{K}=u / K+y .
$$

Como critério de desempenho utilizaremos

$$
I=\int_{0}^{t}\left(y-w_{m} * r_{K}\right)^{2} d \tau
$$

onde $W_{m}(s)$ é uma função de tranferência de comportamento desejável e $w_{m}$ é obtido da transformada inversa de Laplace

$$
w_{m}=\mathcal{L}^{-1}\left[W_{m}(s)\right] .
$$

Introduzimos a notação

$$
\begin{aligned}
& u_{m}=w_{m} * u \\
& y_{m}=w_{m} * y
\end{aligned}
$$

e, consequentemente, teremos

$$
y-w_{m} * r_{K}=y-w_{m} *(u / K+y)
$$

e

$$
\begin{gathered}
I=\int_{0}^{t}\left(y-w_{m} * r_{K}\right)^{2} d \tau=\int_{0}^{t}\left(y-u_{m} / K-y_{m}\right)^{2} d \tau \\
=\int_{0}^{t}\left(y^{2}+\left(u_{m} / K\right)^{2}+y_{m}^{2}-2 y u_{m} / K-2 y y_{m}+2 u_{m} y_{m} / K\right) d \tau \\
=A-2 \frac{B}{K}+\frac{C}{K^{2}},
\end{gathered}
$$

onde

$$
\begin{gathered}
A=\int_{0}^{t}\left(y-y_{m}\right)^{2} d \tau \\
B=\int_{0}^{t}\left(y-y_{m}\right) u_{m} d \tau \\
C=\int_{0}^{t}\left(u_{m}\right)^{2} d \tau
\end{gathered}
$$

Minimizando o critério de desempenho, acharemos o nosso estimador $\hat{K}$ :

$$
\dot{I}=2 \frac{B}{K^{2}}-\frac{2 C}{K^{3}}=0
$$

implica

$$
\hat{K}=\frac{C}{B},
$$

que é o fator de proporcionalidade das funções de controle $u$. O valor de $\hat{K}$ é atualizado dentro de certo período de tempo, para melhorar a convergência. A figura 1 apresenta um diagrama de blocos do controle proposto. 


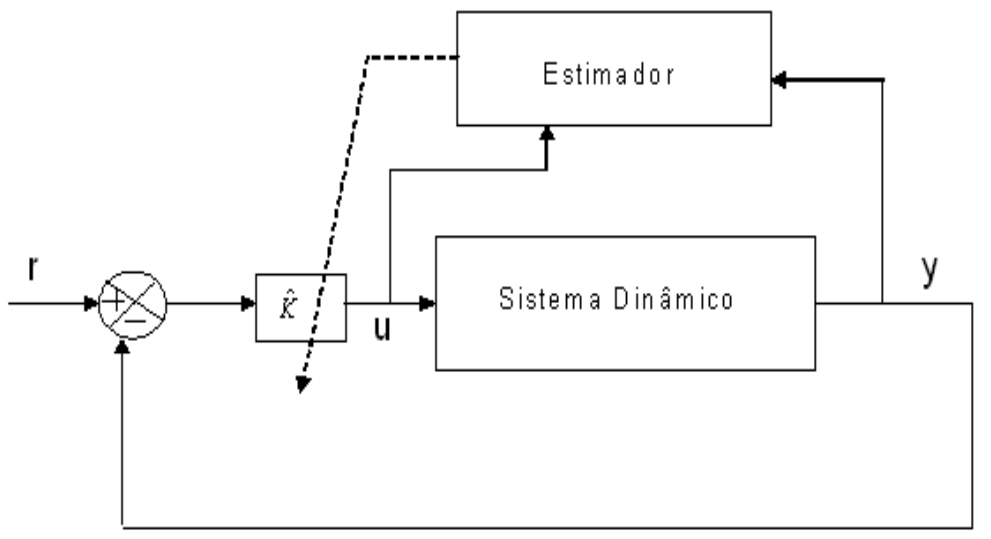

Figura 1: Diagrama de blocos do controle proposto.

\section{Simulações e Resultados}

Nas simulações numéricas foi considerado o sistema dinâmico não-linear de Rössler [3]

$$
\begin{gathered}
\dot{x}_{1}=-x_{2}-x_{3} \\
\dot{x}_{2}=x_{1}+b x_{2} \\
\dot{x}_{3}=b+x_{3}\left(x_{1}-a\right) .
\end{gathered}
$$

Podemos observar que este sistema contém somente uma não-linearidade na terceira equação, $x_{3} x_{1}$. Para os valores de $b=0,2$ e $a=5,7$ o sistema apresenta comportamento caótico.

O sistema controlado proposto é descrito como

$$
\begin{gathered}
\dot{x}_{1}=-x_{2}-x_{3}+u_{1} \\
\dot{x}_{2}=x_{1}+b x_{2}+u_{2} \\
\dot{x}_{3}=b+x_{3}\left(x_{1}-a\right)+u_{3},
\end{gathered}
$$

onde as funções de controle entram no sistema de forma independente em cada equação

$$
\begin{gathered}
u_{1}=K_{1}\left(x_{1}-\tilde{x}_{1}\right) \\
u_{2}=K_{2}\left(x_{2}-\tilde{x}_{2}\right) \\
u_{3}=K_{3}\left(x_{3}-\tilde{x}_{3}\right),
\end{gathered}
$$

sendo uma forma simplificada das funções de controle. No trabalho de [2], as funçoes de controle também contém coeficientes de proporcionalidade, no entanto elas não são independentes nas equações (a função de controle de cada equação depende de todas as variáveis do estado).

Consideramos as trajetórias desejadas como o vetor

$$
\tilde{x}=\left[\begin{array}{lll}
\tilde{x}_{1}(t) & \tilde{x}_{2}(t) & \tilde{x}_{3}(t)
\end{array}\right]^{T}=\left[\begin{array}{lll}
5+\cos (t) & \sin (t) & \sin (t)
\end{array}\right]^{T},
$$

o que em termos de diagrama de fase representa um ciclo limite.

Assim, pela técnica de identificação de controladores temos que o vetor $\tilde{x}$ corresponde a referência fictícia $r_{K}$, eq. (3). O estado $y=\left[\begin{array}{lll}x_{1} & x_{2} & x_{3}\end{array}\right]$ é considerado como entrada de dados do modelo controlado, eq. (16), e as funções de controle são calculadas da equação (17).

Para as simulações foi utilizado o software Matlab e para integração do sistema o método Runge-Kutta de quarta ordem. Os valores dos termos das equações (5)-(13) foram calculados juntamente com o estado, montando um único vetor de funções a serem integradas

$$
y=\left[\begin{array}{llllllllllllllllll}
x_{1} & x_{2} & x_{3} & y_{m}^{1} & u_{m}^{1} & A^{1} & B^{1} & C^{1} & y_{m}^{2} & u_{m}^{2} & A^{2} & B^{2} & C^{2} & y_{m}^{3} & u_{m}^{3} & A^{3} & B^{3} & C^{3}
\end{array}\right]^{T},
$$


onde os índices superiores representam a ordem da equação.

O estado inicial $y_{0}$, os valores iniciais de $K_{1}, K_{2}$ e $K_{3}$ e a função de transferência $W_{m}(s)$ utilizados nas simulações foram

$$
\begin{aligned}
y_{0}=\left[\begin{array}{llllllllllllllllll}
0 & 0 & 0 & 0 & 0 & 0 & 0 & 0 & 0 & 0 & 0 & 0 & 0 & 0 & 0 & 0 & 0 & 0
\end{array}\right]^{T}, \\
K=\left[\begin{array}{llll}
10 & 10 & 10
\end{array}\right]^{T}, \\
W_{m}(s)=\frac{1}{s+1} .
\end{aligned}
$$

O tempo de simulação total foi de $50 \mathrm{~s}$. Os subintervalos para atualização dos valores de $K_{1}$, $K_{2}$ e $K_{3}$ foi de $0,5 s$.

$\mathrm{Na}$ figura 2 são apresentadas as trajetórias desejadas $r_{1}, r_{2}, r_{3}$ e as trajetórias do sistema controlado $x$. É possivel observar que as trajetórias do sistema controlado convergem para as trajetórias desejadas.
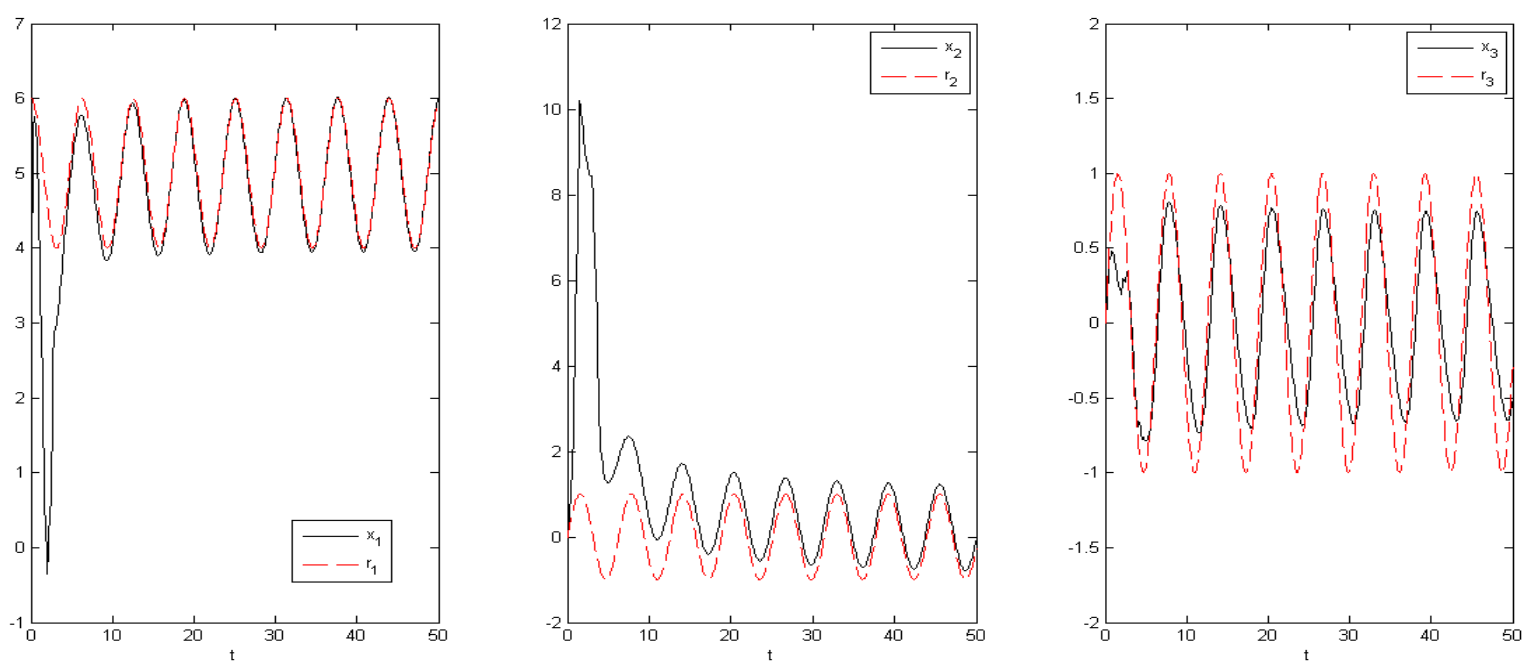

Figura 2: Trajetórias temporais do sistema controlado e trajetórias desejadas.

A simulação do diagrama de fase do sistema de Rössler sem controle é apresentada na figura 3. Já na figura 4 é apresentado o diagrama de fase do sistema de Rössler controlado.

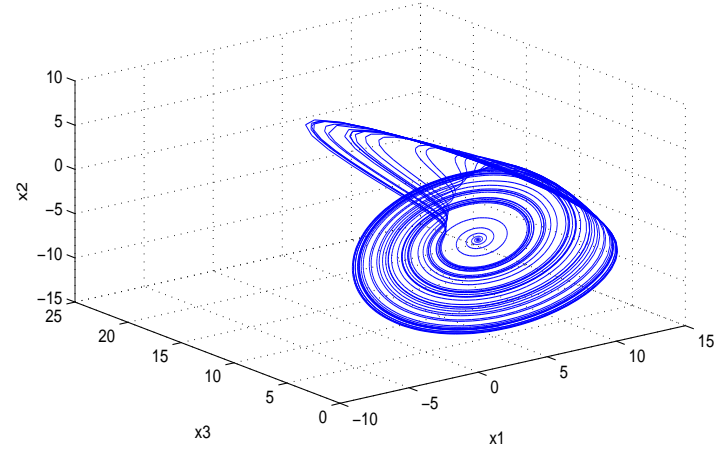

Figura 3: Diagrama de fase do sistema de Rössler.

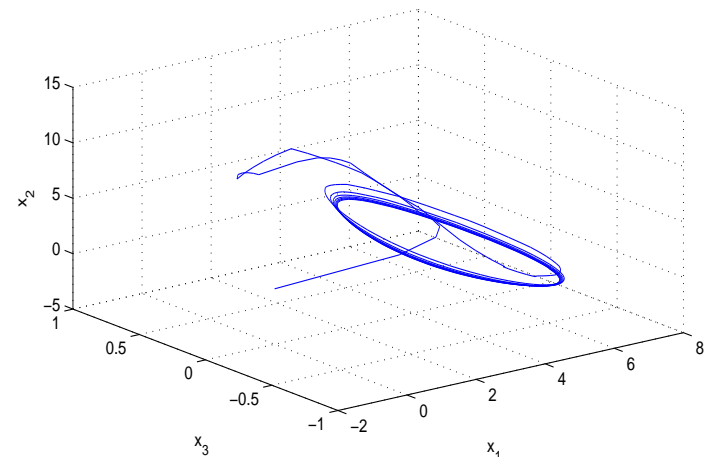

Figura 4: Diagrama de fase do sistema controlado.

Pode-se notar pela figura 4 que há convergência do sistema para o ciclo limite dado por $\tilde{x}$. 


\section{Conclusões}

Neste trabalho foi apresentado um método de identificação de controladores proporcionais para um sistema não-linear sem o conhecimento dos valores dos parâmetros deste e nem das funções de ocntrole. Pelas simulações observou-se que as trajetórias controladas do sistema convergiram para as trajetórias desejadas, o que mostrou a eficiência da metodologia de controle utilizada. A escolha da família de controladores proporcionais, como um exemplo simples, também teve caráter didático, pois mostrou a possibilidade de identificar controladores on-line a partir dos dados experimentais.

\section{Referências}

[1] J. Cassiano, Um Contolador para o sistema de Rössler, In Dincon 2011 - $10^{a}$ Conferência Brasileira de Dinâmica, Controle e Aplicações, (2011) 177-179.

[2] M. Rafikov , J.M. Balthazar, On control and synchronization in chaotic and hyperchaotic systems via linear feedback control, Communications in Nonlinear Science and Numerical Simulation, 13 (2008) 1246-1255.

[3] O. E. Rössler, An equation for continuous chaos, Physics Letters, 57A 5 (1976) 397-398.

[4] M. G. Safonov, F. B. Cabral, Fitting controllers to data, Systems and Control Letters, 43(4) (2001) 299-308.

[5] M.G. Safonov, T. C.Tsao, The unfalsified control concept and learning, IEEE Transactions on Automatic Control, 42 (6) (1997) 843-847.

[6] M.G. Safonov, Origins of robust control: Early history and future speculations, IFAC Proceedings Volumes (IFAC-PapersOnline), 7 (PART 1) (2012) 1-8. 